\title{
Hunger Affects Social Decisions in a Multi-Round Public Goods Game but Not a Single-Shot Ultimatum Game
}

\author{
Sam Fraser $^{1} \cdot$ Daniel Nettle $^{1}$
}

Received: 22 January 2020 / Revised: 17 June 2020 / Accepted: 22 June 2020 /

Published online: 8 July 2020

(C) The Author(s) 2020

\begin{abstract}
Objective People have the intuition that hunger undermines social cooperation, but experimental tests of this have often produced null results. One possible explanation is that the experimental tasks used are not rich enough to capture the diverse pathways by which social cooperation can be sustained or break down in real life. We studied the effects of hunger on cooperation in two tasks of differential interaction richness.

Methods We manipulated hunger by asking participants to eat, or refrain from eating, breakfast. Participants in experiment $1(n=106)$ played a one-shot Ultimatum Game. Participants in experiment $2(n=264)$ played twenty rounds of a Public Goods Game in the same groups of four, ten rounds with the possibility of punishing other group members, and ten without.

Results In experiment 1, skipping breakfast had no significant effects on either amounts proposed or minimum acceptable offers. In experiment 2 , there were multiple different significant effects of the manipulation. No-breakfast participants were more generous in the first round of the game without punishment, and in subsequent rounds, were more influenced by what other group members had done the round before. In the punishment game, no-breakfast participants were also less likely to punish their group-mates than breakfast participants. Consequently, the possibility of punishment was less effective in increasing group cooperation levels in no-breakfast groups.

Conclusion Replicating earlier findings, we found a null effect of hunger on cooperation in a one-shot Ultimatum Game. However, in our richer Public Goods Game, the dynamics of cooperation differed with hunger, in subtle ways not simply classifiable as hungry participants being 'more' or 'less' cooperative overall.
\end{abstract}

Keywords Cooperation · Hunger - Altruistic punishment · Economic game · Decision latency

Daniel Nettle

daniel.nettle@ncl.ac.uk

Extended author information available on the last page of the article 


\section{General Introduction}

People have a clear intuition that hunger undermines social cooperation. For example, in a recent survey study, $73 \%$ of participants stated that they believe hunger makes people less cooperative (Häusser et al. 2019; study 5). The anthropological literature is full of accounts of 'famine crime' (Arnold 1993); chronic hunger curtailing all generosity (Richards 1932); or individuals becoming 'atomistic' and 'egotistical' when food is short (Turnbull 1972). These accounts seem intuitively compelling, though an oddity is that the literature also contains many claims of hunger apparently bringing people together to share (Dirks 1980).

Given the generality of the belief that hunger must undermine cooperation, it is not surprising that researchers have studied the effects of short-term hunger manipulations on laboratory cooperation paradigms, usually with the expectation that the hungry participants will cooperate less than controls. Though some studies report results conforming to expectation (Briers et al. 2006; Xu et al. 2014; Harel and Kogut 2015), other results have been null. Hausser et al. (2019) either manipulated or measured hunger prior to a series of different cooperation tasks and measures (economic games, survey measures and a volunteering task), finding no significant hunger effects on any outcome. Similarly, Rantapuska et al. (2017) administered a series of economic games and cooperative decisions to overnight-fasted participants who had been given either a standardized meal or not prior to the session. Hungry participants were significantly more cooperative in a prisoner's dilemma, and tended to be more cooperative in the sender role of a trust game, whilst donations to charity, contributions in a public goods game, and behaviour in the sender role of the trust game were not significantly different. Thus, the corrosive effect of hunger on cooperation that people intuitively feel should be present, and the anthropological literature suggests may often exist when hunger is widespread and chronic in society, does not reliably show up when acute hunger is manipulated in the experimental psychology laboratory.

One possible reason for the gulf between the lay belief and anthropological literature on the one hand, and the experimental results on the other, is that the experimental paradigms used do not capture the relevant features of real-world social cooperation. One can think of laboratory cooperation tasks as being on a continuum of interaction richness. At one end of the continuum are one-shot, non-interdependent paradigms such as the Dictator Game (DG) where the actor's outcome does not depend on the decisions of others, and there are no social consequences possible within the game. Inter-dependent tasks such as the Ultimatum Game (UG) are somewhat richer, in that the proposer's outcome is affected by the behaviour of the responder. Richer still are paradigms like the multi-round Public Goods Game (PGG) with punishment. Here, participants can respond to the previous behaviour of other group members; can choose to actively sanction others; can respond to being sanction by their behaviour in future rounds, and so on.

The experimental effects of hunger on cooperation have been most studied in tasks towards the less rich end of the continuum. Many of these are DG or unconditional donation-style tasks (Briers et al. 2006; Xu et al. 2014; Harel and Kogut 2015), whilst Rantapuska et al. (2017) used two tasks that were interdependent, but where there was no possibility for one player to sanction the other and no repeated interaction between the same players. Häusser et al. (2019) extended the set of tasks studied to include the 
Ultimatum Game. Here, the second player (the responder) has the possibility of sanctioning the first player (the proposer), by refusing a low offer even though this will be costly to themselves. However, even in the study by Häusser et al. (2019), there was no repeated interaction between the same players, and hence no possibility of rich interpersonal dynamics.

Which kind of task is adequate for experimentally modelling the likely real-world effects of hunger on cooperation depends upon one's expectations about what mechanisms might underlie those effects. For example, if hunger has a strong direct negative effect on prosocial motivation, then a one-shot DG-style task should show it clearly. Current support for the existence of such a direct effect is weak (Häusser et al. 2019). However, even in the absence of such a direct effect, there are other pathways by which hunger could cause the results of cooperative endeavours to turn out differently in the real world. For example, hungry people might have the same initial prosocial motivations as non-hungry people, but respond more negatively or less forgivingly to the behaviour of others. This possibility is supported by large literatures showing that hunger increases irritability (see Nettle 2017). This would suggest a more rapid 'race to the bottom' in complex cooperative tasks where the interactants are hungry, especially once at least one group member is not cooperating fully. On the other hand, Nettle (2019) hypothesizes that, when hungry, people might switch to imitating others rather than extensive individual computation of costs and benefits, since such computation is metabolically expensive. This would predict that hungry groups would be more likely than non-hungry groups to converge on a shared norm. This norm need not necessarily be a non-cooperative one, however; it could be either cooperative or non-cooperative, depending on members' initial behaviour.

In real-world settings, cooperation may be sustained by punishment, namely interventions to impact others at cost to oneself. Punishment is thought to be important in maintaining human cooperation over time in realistic scenarios (Boyd and Richerson 1992; Fehr and Gächter 2002). Hungry people could use punishment differently. Here, previous findings would licence predictions in either direction. On the one hand, punishment can be seen as a form of aggression, and aggression is generally increased by hunger, or low blood glucose (DeWall et al. 2011; Bushman et al. 2014). This suggests the prediction that there would be more punishment when people are hungry, and hence possibly more cooperation, though this would depend on whether the increased punishment under hunger was effectively directed at non-cooperators, or devolved into antisocial or revenge punishment (Raihani and Bshary 2019). On the other hand, recent studies have found that hunger reduces moral disapproval (Vicario et al. 2018), and leads people to suggest more lenient punishments for moral violations (Kerry et al. 2019). The authors of these studies speculate that hunger down-regulates disgust, an emotion important in regulating food intake, but also in moral decisionmaking. These findings suggest that hungry people would punish less, again possibly leading to less cooperation.

In short, there are a number of pathways by which the outcomes of real-world cooperative endeavours might end up different when participants are hungry compared to not hungry. These pathways are generally not captured by the very simple cooperation tasks used in the experimental literature so far. Though these pathways could lead cooperation to break down under hunger, as the lay belief suggests, they could under certain circumstances lead to more cooperation when people are 
hungry. Predictions are possible in either direction, and it is not clear a priori which pathway might be the most important.

In this paper, we report the results of two experiments in which we manipulated hunger and studied the effects on cooperative behaviour. Our overall aim was to extend the interactional richness of the experimental literature on hunger and cooperation, and hence explore the possibility of effects of hunger on cooperative behaviour that would not be detectable in a simple DG. In study 1, we introduced the possibility of punishment, of a kind, by using a UG (Guth et al. 1982). At the time of conception, this was novel. However, in the interim, the paper by Häusser et al. (2019) appeared, and so our experiment 1 became a replication of their study 2 . In experiment 2, we used a multi-round PGG with punishment (Fehr and Gachter 2000). This incorporates much richer behavioural possibilities than any previous paradigm.

Note that our two experiments differ from one another in multiple ways. The UG is usually considered a test of preference for fairness within dyads, whilst the PGG is thought of as a way of studying the factors affecting the ability of groups to maintain public goods. The UG of experiment 1 includes a possible sanction of the proposer by the responder, but this is not framed as punishment as such. Moreover, as there was only one round, there was no potential to change the other player's behaviour within the game. The two parameters that can be measured from the UG are the proposer's offer, which may reflect both their prosociality and their inferences about the likely behaviour of the responder; and the responder's minimum acceptable offer, which can be considered their preference for fairness or willingness to punish unfairness. By contrast, the PGG in experiment 2 allows separate measurement of the participant's initial offer (which might be a considered a simple measure of prosocial motivation), their response in later rounds to what others did in earlier ones, their propensity to punish, and their response to being punished. (Fehr and Gachter 2000; Gunnthorsdottir et al. 2007). Hunger could have separate effects on any these, resulting in different evolution of group public goods over time.

We did not pre-register predictions for either experiment. Although we were driven by the standard hypothesis that hunger will lead to less cooperation, as we have discussed above, there are several variables where it is possible to make predictions for the effect of hunger in either direction. We therefore present the results as exploratory, and conclusions as suitably tentative. We also present a systematic comparison of the results of the two experiments, following the individual results presentations.

\section{Experiment 1: Introduction}

Experiment 1 was performed after experiment 2, but they are presented in this order for clarity of logic. Experiment 1 used a single-shot UG. In the UG, one participant proposes a split of a monetary endowment between themselves and another participant. The responder either accepts the split, in which case the money is paid, or rejects it, in which case both participants receive nothing. We, however, used the strategy method, whereby the responders specifies, for every possible offer the proposer might make, whether they would accept or reject. The income-maximising strategy for the responder is to accept any non-zero offer. The income-maximising strategy for the proposer is to propose the lowest offer likely to be acceptable to the responder. In practice, low but non-zero offers 
are typically rejected, and responders offer more than they would need to have their offers accepted (Oosterbeek et al. 2004; Tisserand et al. 2015). Hausser et al. (2019; study 2) randomly assigned overnight-fasted participants to either complete the UG hungry, or receive food first. They found no significant hunger effect for either role.

We manipulated hunger by asking participants to either skip breakfast on the day of the experimental session, or to eat breakfast as usual. We have used this manipulation before, and shown it to be effective in producing differences in impulsivity as well as self-reported hunger (Allen and Nettle 2019). All players completed UGs both as proposer and responder, and we report the effects of the no-breakfast manipulation on both decisions. As we did not pre-register predictions, and were unaware of the results of Hausser et al. (2019; study 2) when we performed the study, we present our analyses as exploratory.

In addition to the main measures, we collected data on participants' latency to decide, and also a cognitive reflection test (CRT). This is a widely used measure of deliberate versus intuitive decision-making. In many of the cooperation tasks used by psychologists and behavioural economists, a quick, intuitive decision tends to favour more cooperation, since participants then use simple heuristics derived from real-life social encounters (Rand 2016). It is participants who deliberate more extensively about the specific payoff structure of the game who realise that cooperation is not the incomemaximising strategy. Hungry participants tend to use faster or more intuitive styles of decision-making (Orquin and Kurzban 2016). Thus, as Rantapuska et al. (2017) point out, we might expect hungry participants to be more cooperative, through deliberating less. If this were the case, we should be able to detect experimental effects on decision latency and cognitive reflection, and associations between decision latency and cognitive reflection and cooperative decisions in the $\mathrm{UG}$.

\section{Methods}

\section{Participants}

We recruited 106 participants from registers held at Newcastle University (66 male, 40 female). Participants were mostly students. Due to use of genuine randomization to assign participants to conditions, our experimental groups were not equal in size (breakfast, 60; no-breakfast 46). A compromise power analysis using GPower (Faul et al. 2007) indicated that these group sizes would give us $77 \%$ power to detect a medium-sized $(d=0.5)$ simple experimental effect. All participants gave written informed consent to participate. Participants attended singly and made their decisions at a computer with the experimenter withdrawing. They received a $£ 5$ gift voucher as a show-up fee, in addition to whatever money they took away from the UG. The experiment was approved by the Faculty of Medical Sciences ethics committee, Newcastle University.

\section{Ultimatum Game}

Each participant completed both the proposer and responder role of the UG, with the order counterbalanced. Game rules were fully explained prior to the first role, and the 
participant was given a chance to ask questions. The decisions of the previous participant were used to decide game outcome. Information on the other player's decisions was not provided until both proposer and responder decisions had been made. At the end of the session, money from one of the two roles was paid out according to game decisions, a visible coin toss deciding which one.

For the proposer role, the participant chose an amount to offer by moving a bar on a computer screen anywhere from $£ 0$ to $£ 10$ in increments of $£ 0.50$. The responder role used the strategy method: participants were provided with a list of all the 21 possible offers the proposer might make, and indicated which ones they would accept. From this, we calculated the participant's minimum acceptable offer.

\section{Cognitive Reflection Test}

Participants completed a version of the CRT after completing the UG. We used an 8-item test, using the original three items introduced by Frederick (2005), four further items from the expanded version of the test (Toplak et al. 2014), and an additional item featured in Trouche, Sander and Mercier (2014). Each item consists of a question (e.g. 'If it takes 5 machines 5 minutes to make 5 widgets, how long would it take 100 machines to make 100 widgets?') with intuitively obvious but wrong answers (e.g. $100 \mathrm{~min}$ ), and a correct answer ( $5 \mathrm{~min}$ ) that requires more deliberation. The score is the number of correct items, which should reflect the degree of deliberation an individual is using.

\section{No-Breakfast Manipulation}

Participants were invited to take part in the study via email. This email instructed them to either eat as normal, or refrain from eating, breakfast on the morning of the session. Self-reported compliance was collected in an initial questionnaire, as was self-reported hunger on a ten-point scale.

\section{Data Analysis}

Data were analysed using general linear models in R (R Core Development Team 2018). Our main outcome variables were the proposer amount offered, and the responder minimum acceptable offer. Secondary variables were the latency to decide in both roles, and scores on the CRT. Experimental effects were primarily analysed on an intention to treat basis. That is, we used as our explanatory variable the condition to which the participant was assigned. However, since compliance with the instructions was not perfect (see Results), we also reran all analyses using self-rated hunger as the explanatory variable; none of the results reported below was affected. Decision latencies were log-transformed.

\section{Results}

\section{Manipulation Checks}

Of the participants assigned to the breakfast condition, $75 \%$ reported that they had in fact eaten breakfast. Of those assigned to the no-breakfast condition, 
95.7\% reported that they had in fact skipped breakfast. Participants in the nobreakfast condition reported being significantly hungrier (mean 6.13, s.d. 2.06) than those in the breakfast condition (mean 3.23, s.d. 2.29, $\mathrm{t}=6.73, p<0.001$, $\mathrm{d}=1.33$ ). There were no significant differences in amount offered or minimum acceptable offer according to the order the two UG roles were played in (amount offered: first, 4.82, s.d. 1.87; second, 4.84, s.d. 1.29; $\mathrm{t}=0.06, p=$ $0.95, \mathrm{~d}=0.01$; minimum acceptable offer: first, 3.50, s.d. 1.57; second, 3.32, s.d. $1.61 ; \mathrm{t}=0.57, p=0.57, \mathrm{~d}=0.11)$. There was a marginally non-significant, weak negative correlation between participants' proposed amounts and their minimum acceptable offers $(r=-0.18 . p=0.06)$.

\section{Experimental Effects: Proposer Role}

There was no significant effect of condition on amount offered in the proposer role ( $\mathrm{B}=0.11$, s.e. $0.32, \mathrm{t}=0.33, p=0.740$ ), though the mean was slightly higher for the nobreakfast condition (breakfast: 4.78 , s.d. 1.33; no breakfast: 4.89, s.d. 2.01). The distribution of offers was dominated by a mode of $£ 5$ (i.e. $50 \%$ of the stake; Fig. 1a). This constituted $42 \%$ of all offers. Dividing offers dichotomously into those of $50 \%$ of the stake or more, and those less than $50 \%$ of the stake, the association of offer type with condition was not significant $\left(\chi^{2}=0.85, p=0.36\right)$. Amongst breakfast participants, $57 \%$ offered $50 \%$ of the stake or more; amongst no-breakfast participants, $67 \%$ did.

\section{Experimental Effects: Responder Role}

There was no significant difference in minimum acceptable offer by condition $(\mathrm{B}=$ 0.32 , s.e. $0.32, \mathrm{t}=1.02, p=0.31$ ). The mean minimum acceptable offer was slightly higher for the no-breakfast condition (breakfast: 3.26, s.d. 1.53; no breakfast: 3.58, s.d. 1.66). This was driven by a non-significant trend for more responders to demand fully $50 \%$ of the stake in the no breakfast condition (35\% of responders in no breakfast condition; $20 \%$ in breakfast condition; $\chi^{2}=2.22, p=0.14$; Fig. 1b).
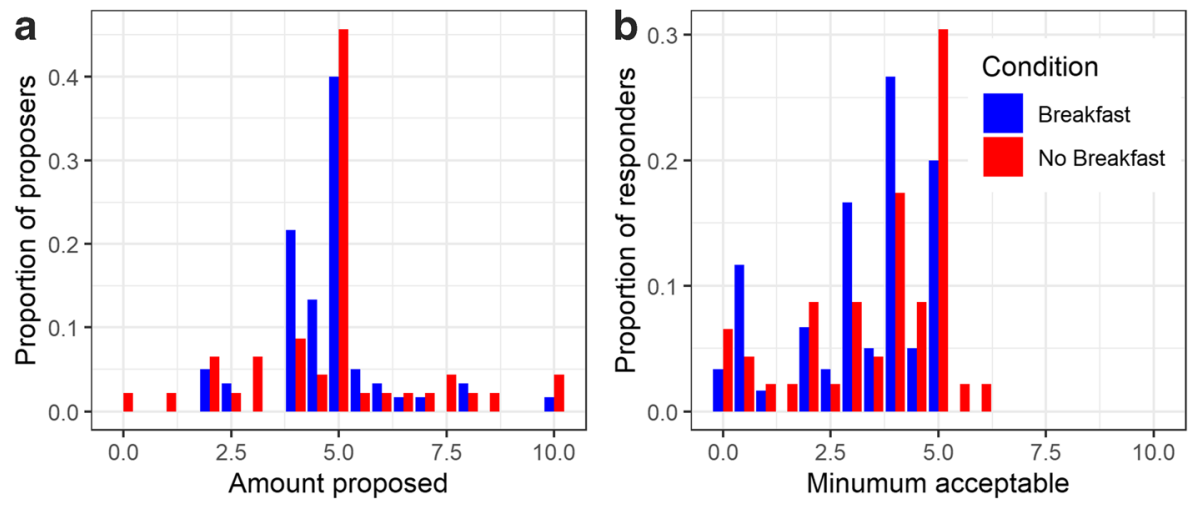

Fig. 1 Experiment 1 results. a Distribution of amounts proposed in the UG, by experimental condition. b Distribution of minimum acceptable offers, by experimental condition. $N=106$ (60 in the breakfast condition, 46 in the no-breakfast condition) 


\section{Decision Latency}

There was no effect of condition on decision latency in either the proposer role (breakfast: $3.26 \log$ seconds, s.d. 0.57 ; no breakfast: 3.24 , s.d. $0.44 ; \mathrm{t}=-0.18, p=$ 0.86 ), or the responder role (breakfast: 4.21 , s.d. 0.45 ; no breakfast: 4.28 , s.d. 0.47 ; $\mathrm{t}=$ $-0.75, p=0.45)$. In the proposer role, there was a significant though weak positive correlation between decision latency and amount proposed $(\mathrm{r}=0.21, p=0.03)$. In the responder role, the association between decision latency and minimum acceptable amount was not significantly different from zero $(\mathrm{r}=0.02, p=0.85)$.

\section{Cognitive Reflection Test}

The effect of condition on number of CRT questions correct was not significant (breakfast: 3.73, s.d. 2.07; no breakfast: 3.26, s.d. 2.00; $\mathrm{t}=-1.18, p=0.24$ ). Moreover, the no-breakfast participants did not spend any less time on the CRT questions than the breakfast participants did (breakfast: 6.05, s.d. 0.46; no breakfast: 6.13 , s.d. 0.45 ; $\mathrm{t}=$ $0.97, p=0.33)$. Number of CRT questions correct was not significantly associated with amount offered in the proposer role $(\mathrm{r}=0.05, p=0.65)$, or minimum acceptable offer in the responder role $(\mathrm{r}=-0.15, p=0.13)$.

\section{Experiment 1 Discussion}

Using a one-shot UG, we found no clear evidence that skipping breakfast had any effect on either the amount proposed or the minimum acceptable offer. Thus, we reproduce the null results of a similar recent study (Häusser et al. 2019; study 2). There was considerable variation in behaviour in both roles (Fig. 1). Although the modal amount offered was $£ 5.00$, only a minority of participants chose this offer. Moreover, the no-breakfast manipulation was effective in increasing self-rated hunger. Thus, the experimental manipulation was effective, and there was variability in the outcome, so there was scope for detecting a hunger effect if one exists.

As well as the non-significant experimental effects on the main outcomes, we did not find a number of other relationships that we might have expected. If hunger causes people to adopt a more intuitive, heuristic approach to social decision making, then we expected it to make decision latencies faster, and scores on the CRT lower. The experimental treatment had neither of these effects. Moreover, there were no relationships between CRT score and UG behaviour. The amount offered as proposer actually increased slightly with increasing time taken to decide. This is the opposite to the prediction stated in Rand (2016), namely that in this one-shot game, more deliberation should be associated with lower offers. However, we would note that the UG has a structural ambiguity. Although the behaviour of the proposer is often interpreted as representing prosocial motivation, it could also be affected by risk aversion. To offer less, given uncertainty about the behaviour of the responder, is to risk coming away with nothing. More deliberation may have led proposers to appreciate the greater riskiness of making a low offer. Rand's (2016) prediction appears about deliberation leading to low offers seems better applicable to the simpler DG, where offering less entails no risk. 
In summary, a simple one-shot UG here revealed no significant effects of breakfastskipping on either the amount offered to another party, or the minimum acceptable offer. As stated in the General Introduction, though, there may be more potentially for revealing effects of hunger on cooperation using a task with repeated interactions between the same individuals over time.

\section{Experiment 2: Introduction}

Experiment 2 replicated the classic PGG study of Fehr and Gachter (2000), with an additional experimental manipulation of hunger. Participants, in groups of four, played a sequence of ten rounds of a PGG with no possibility of punishment, and ten rounds where they have the option to sanction one another on the basis of contributions to the central pot. Experimental sessions were conducted in the morning, and, as for experiment 1 , the experimental manipulation was the instruction to either breakfast as usual (breakfast condition), or skip breakfast (no breakfast condition). The canonical finding in PGG experiments of this type is that, in the no-punishment game, contributions to the central pot are in the vicinity of $50 \%$ of endowment in the first round, but successively reduce over the rounds, approaching zero in many groups by ten rounds. By contrast, in the punishment game, contributions are stable and may even increase over the course of the rounds.

The data from experiment 2 allow separate estimation of the effects of hunger on a number of different variables, all within the same dataset. These include: the contribution in the first round of the no-punishment game; the magnitude of players' responses in subsequent rounds to what others had done the round before; the propensity to punish others; and the response to being punished. As we outlined in the General Introduction, there are reasons for expecting that hunger could influence several of these variables, but in some cases predictions could plausibly be made in either direction. We did not preregister predictions and present the results below as exploratory.

\section{Methods}

\section{Participants}

A total of 264 participants were recruited from registers held at Newcastle University (132 in sample 1, 132 in sample 2; 147 female and 117 male). Participants were mostly students. The two samples did not overlap. Participants were paid a $£ 2$ participation fee, and in addition, credits accumulated in the PGG were converted to cash at the rate of $£ 1$ per 50 credits. Game earnings averaged 394 credits (i.e. £7.88). All participants gave written informed consent to participate. The experiment was approved by the Faculty of Medical Sciences ethics committee, Newcastle University.

\section{Public Goods Game}

A four-player multi-round PGG, with both a punishment and no-punishment version, was implemented in oTree (Chen et al. 2016). Participants completed the game on 
networked computers in a room with 36 individual desks with separation screens. At least 8 participants were present per session and participants did not know which others were members of their game group. Within each round, each player was given an initial endowment of 20 credits, and had to decide how much, if any, to allocate to a central pot. Allocations to the central pot were multiplied by 1.4 , and the pot was divided equally between the four group members. In the punishment version of the game, following decisions about how much to contribute to the central pot, which were known to all players, participants could elect to use their credits to reduce the earnings of other group members. Each credit used for this purpose decreased the income of the selected individual by $10 \%$ for the round, to a minimum of zero. Credits were accumulated across rounds for final conversion into cash. Participants completed 10 rounds of the no-punishment game, and 10 rounds of the punishment game, with the order of the two game versions counterbalanced. Group composition remained the same for all 20 rounds.

All game information was explicitly presented in initial on-screen instructions. Prior to the first round, participants completed three multiple-choice questions to demonstrate understanding of game rules for the payoffs from the common fund, and an additional two in the punishment game to demonstrate an understanding of how punishment worked. Comprehension questions used a multiple-choice response format. On completion of the final round, participants remained at their desks and were brought a sealed envelope containing the conversion of their accumulated credits to cash.

\section{No-Breakfast Manipulation}

Participants were invited to take part in the study via email, one to three days before the session. This email instructed them to either eat as normal, or refrain from eating, breakfast on the morning of the session. Self-reported compliance was collected in an initial questionnaire, as was self-reported hunger on a ten-point scale. The experimental treatment was randomised on a session level: that is, all participants on a given day, and therefore all members of each game group, were in either the breakfast or no-breakfast condition.

\section{Data Analysis}

The primary outcome measures were contribution to the central pot (0-20), and punishment allocated (punishment game only). Latency to make each decision (seconds, from the decision screen appearing to the participant entering their choice) was also recorded, and we used this as an ancillary outcome measure. Due to a server problem, latency data were only available for 220 of the 264 participants ( 88 of sample 1, all 132 of sample 2). Experimental effects were analysed on an intention-to-treat basis. That is, the independent variable was the treatment the participant had been assigned to, rather than whether they had in fact eaten breakfast or not.

Data were analysed in R (R Core Development Team 2018) using general linear and, where appropriate, linear mixed and generalized linear mixed models to take account of the structure of the data (repeated rounds within participant, and participants clustered in groups). Linear mixed models were fitted using maximum likelihood estimation. Significance tests for parameters in mixed models used Satterthwaite's method via R package 'lmerTest'. 
We first fitted a simple model of the overall pattern of contributions (model 1; full output for all models is shown in Appendix Table A1). The a priori fixed predictors were: game (punishment vs. no punishment) and number of previous rounds $(0-9$. We expected, based on previous literature, an interaction between game and number of previous rounds, with contributions declining over the rounds in the no punishment game, but not the punishment game. We included experimental condition and all its interactions (with round and game) in our model 1. Since our aims were exploratory, we had open predictions about whether experimental effects would appear in the main effect or interaction terms, and about their direction.

We followed up the global results of model 1 with more detailed analyses of each game. For the no-punishment game, we modelled the effect of condition on amount contributed in the first round (model 2). We then fitted a model on the data from round 2 onwards, with contribution as the outcome variable, and as predictors the participant's contribution in the previous round, the average contribution of the other group members in the previous round, condition, and the interaction of condition with the average contribution of other players (model 3). For the punishment game, we modelled the decision to punish in relation to condition and the contributions offered by other group members (model 4). The decision to punish was treated as binary (punish at least one group member vs. not punish), and hence used a generalized linear mixed model with binomial error structure. Finally, we modelled the response to punishment (model 5). Here, the outcome variable was contribution, and the predictors were the participant's contribution in the previous round, whether they were punished in the previous round, condition, and all interactions of these predictors.

In ancillary analyses, we also examined latency to decide for both contributions and (in the punishment game) punishment (models 6 and 7). Latencies to decide were logged for analysis.

\section{Results}

\section{Manipulation Checks}

Of the participants assigned to the breakfast condition, $84.6 \%$ reported at the experimental session that they had in fact eaten breakfast. Of those assigned to the no-breakfast condition, $99.2 \%$ reported that they had in fact skipped. Participants in the no-breakfast condition reported being significantly hungrier (mean 5.59, s.d. 2.18) than those in the breakfast condition (mean 3.12, s.d. $2.18 ; \mathrm{t}=9.16, p<0.001, \mathrm{~d}=1.13$ ). At the group level, 31 groups in the nobreakfast condition contained four members who had not had breakfast, and one group contained three who had not and one who had. In the breakfast condition, 18 groups contained four participants who had had breakfast; 12 groups contained three; three groups contained two; and one group contained one. Repeating the analyses reported below but using the number of participants in the group who had actually had breakfast produces essentially the same results. 


\section{Game Comprehension Questions}

Comprehension questions were not all answered correctly (mean central pot questions: 4.21 out of 6 , s.d. 1.67; mean punishment questions: 1.32 out of 2 , s.d. 0.79 ). The number of central pot questions correct predicted contributions: those who answered more questions correctly contributed less (linear mixed model with game, number of previous rounds and number of central pot questions correct as predictors: $\mathrm{B}=-0.31$, s.e. $0.11, \mathrm{t}=-2.97, p=0.003$ ). Likewise, those who answered more punishment questions correctly were less likely to punish (generalized linear mixed model with mean contribution of others plus punishment questions correct: $\mathrm{B}=-0.44$, s.e. 0.11 , $\mathrm{z}=-3.80, p<0.001)$.

However, comprehension questions correct did not differ significantly by condition (central pot: breakfast: 4.18 , s.d. 1.72 ; no breakfast: 4.24 , s.d. $1.62 ; \mathrm{t}=-0.32, p=0.749$, $\mathrm{d}=-0.04$; punishment: breakfast: 1.27 , s.d. 0.76 ; no breakfast: 1.37 , s.d. $0.81 ; \mathrm{t}=$ $-0.98, p=0.329, \mathrm{~d}=-0.13$. None of the results reported below for experimental condition is affected by the inclusion of number of comprehension questions correct as an additional predictor variable.

\section{Overall Patterns of Contribution}

The overall pattern of contributions, in relation to game, condition, and round, was as shown in Fig. 2a. The expected basic pattern - contributions declining over the rounds in the no-punishment game, but not in the punishment game, was clearly seen. In our model of the overall pattern of contributions (Appendix Table 1, model 1), there was a significant main effect of condition $(\mathrm{B}=1.96$, s.e. $0.90, \mathrm{t}=2.17, p=0.033)$. This suggests that participants in the no-breakfast groups contributed more in the first round of the no-punishment game than those in the breakfast group. The interaction between number of previous rounds and condition was not significant $(\mathrm{B}=-0.12$, s.e. $0.07, \mathrm{t}=$ $-1.76, p=0.079)$. However, there was a significant interaction between game and condition $(\mathrm{B}=-1.42$, s.e. $0.52, \mathrm{t}=-2.75, p=0.006)$. This suggests that the possibility of punishment was less effective in increasing contributions in the no-breakfast groups than the breakfast groups. This is evident in Fig. 2a: the gap between the punishment and no-punishment lines is smaller for the no-breakfast groups than the breakfast groups. The three-way game by condition by number of previous rounds interaction was marginally non-significant $(\mathrm{B}=0.18$, s.e. $0.10, p=0.07)$.

\section{Detailed Analyses: No-Punishment Game}

For the data from the no-punishment game, we first modelled condition effects on contributions in the first round (model 2). First-round contributions were significantly higher in the no-breakfast groups (mean 10.20, s.d. 5.79) than the breakfast groups (mean 8.26, s.d. 6.06; $\mathrm{B}=1.94$, s.e. $0.73, \mathrm{t}=2.66, p=0.008$; Fig. $2 \mathrm{~b}$ ). When then modelled, for subsequent rounds, how a participant's contribution was affected by the mean contribution of other group members in the previous round, condition, and their interaction (model 3). There was a significant interaction between condition and the lagged contribution of other group members $(B=0.14$, s.e. $0.05, t=2.70, p=0.007)$. Participants in the no-breakfast groups were more strongly influenced than those in the 

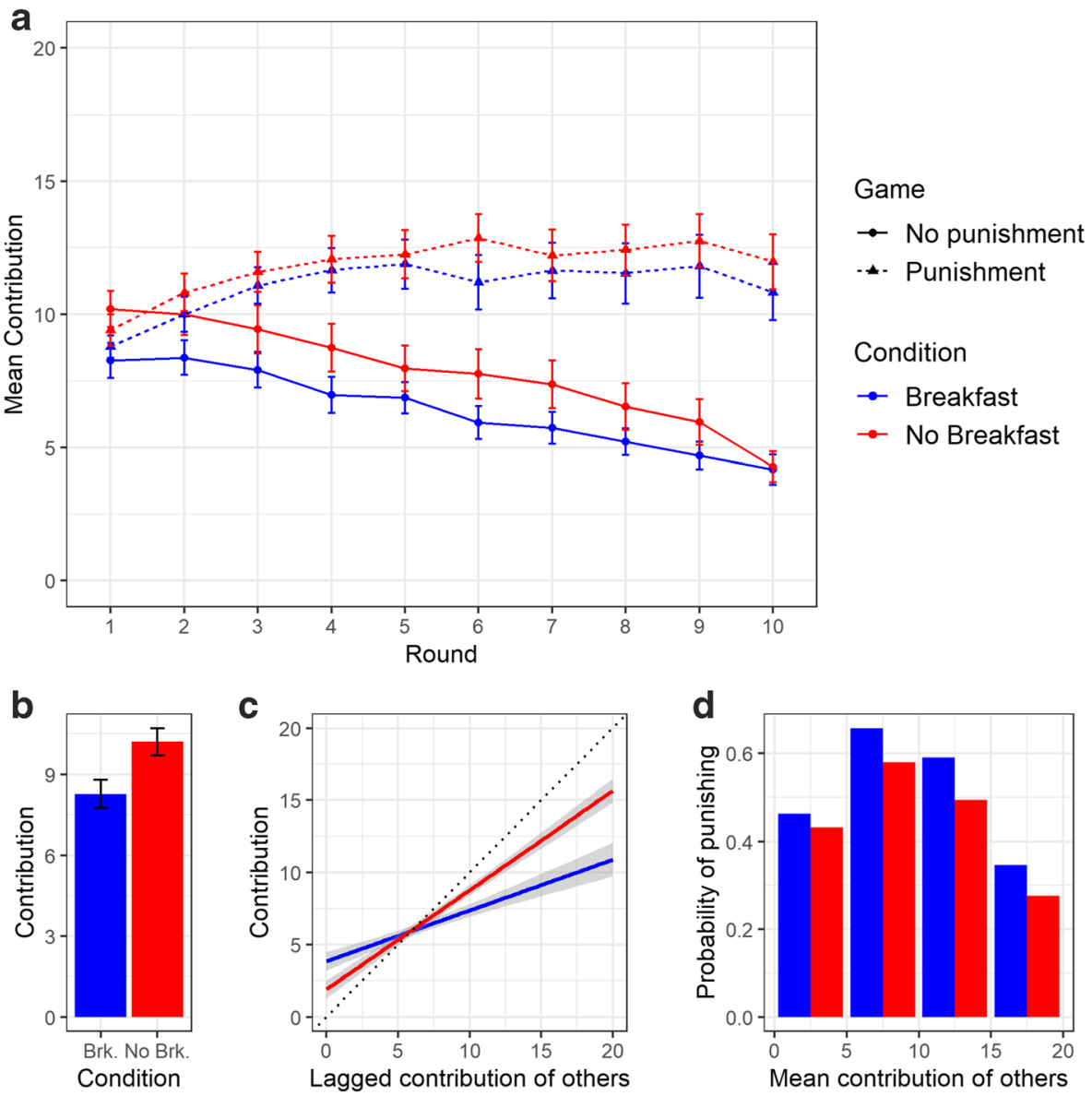

Fig. 2 Experimental effects in experiment 2. a Mean contribution per round, by game (solid lines: no punishment; dotted lines: punishment) and condition (blue: breakfast; red: no breakfast). Errors represent one between-group standard error. b Mean contribution on first round of no-punishment game, by condition. Error bars represent one standard error. $\mathbf{c}$ Individual contribution in relation to the mean contribution of other group members in the previous round, non-initial rounds of no-punishment game, by condition (blue: breakfast; red: no breakfast). The dotted line would represent perfectly matching contributions to the previous group mean. Lines represent linear fits from the data, and shading, 95\% confidence intervals. d Probability of punishing by mean contribution of other group members, and condition (blue: breakfast; red: no breakfast), punishment game

breakfast groups by the previous-round contributions of other group members, contributing less when others had contributed less, but more when others had contributed more (Fig. 2c).

\section{Detailed Analyses: Punishment Game}

For the punishment game, we examined the decision to punish other group members in relation to the contributions offered, condition and their interaction (model 4). There was a significant interaction between condition and the contributions made by others $(\mathrm{B}=-0.52$, s.e. $0.16, \mathrm{z}=-3.14, p=0.002)$. Participants in the no-breakfast groups were 
less likely to punish, especially when the contribution levels were high (Fig. 2d). Overall, in the breakfast groups, participants chose to punish at least one other group member on 717 of 1360 possible occasions (52.7\%). In the no-breakfast group, they chose to punish on 575 of 1280 possible occasions $\left(44.9 \% ; \chi^{2}=15.74, p<0.001\right)$. Finally, we examined the response to being punished, in terms of contribution the next round (model 5). There was a significant interaction between own previous-round contribution and being punished $(\mathrm{B}=0.17$, s.e. $0.05, \mathrm{t}=-3.35, \mathrm{p}<0.001$; people who made high contributions and got punished reduced them, whilst those who made low contributions and were punished increased them). However, none of the effects involving condition was significant. Thus, there was no evidence that people in the nobreakfast groups responded any differently to being punished.

\section{Effects of Condition on Decision Latency}

We examined, for the subset of participants where latency data were available, the latency to decide how much to contribute, in relation to number of previous rounds, game and condition (model 6; Fig. 3). There was a significant main effect of condition ( $\mathrm{B}=-0.21$, s.e. $0.06, \mathrm{t}=-3.62, p<0.001)$. There was also a significant interaction between game and condition $(\mathrm{B}=0.30$, s.e. $0.07, \mathrm{t}=4.52, \mathrm{p}<0.001)$; a shorter average decision latency for no-breakfast participants was seen in the no-punishment game (breakfast: mean $10.60 \mathrm{~s}$, s.d. 30.4; no breakfast: mean 7.40 s, s.d. 7.99), but not the punishment game (breakfast: mean 9.34 s, s.d. 11.60; no-breakfast: mean 10.10, s.d. 12.00).

The effect of condition on decision latency in the first round of the nopunishment game did not mediate the effect of condition on contributions, since there was only a negligible correlation between decision latency and amount contributed $(\mathrm{r}=-0.06, p=0.379)$.

We also examined, for the punishment game, the latency to decide whether to punish other group members or not (model 7). Neither the main effect of condition, nor its interaction with number of previous rounds, was a significant predictor.

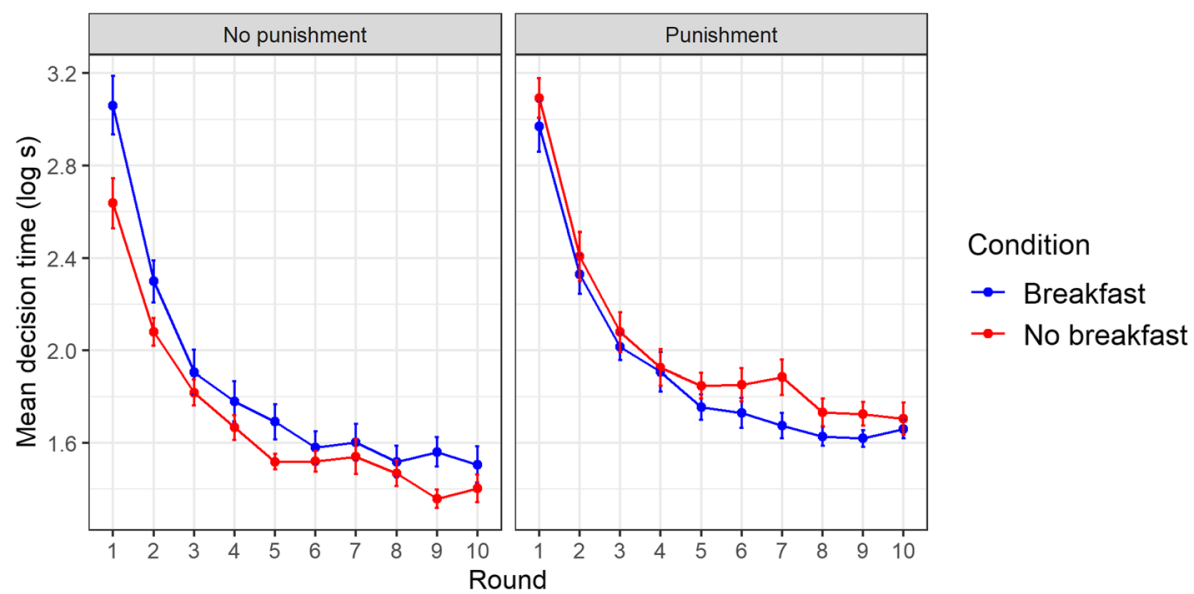

Fig. 3 Latency to decide on how much to contribute by round, game and condition, experiment 2. Shown are means and between-group standard errors of the group mean 


\section{Discussion}

In experiment 2, we found that a breakfast-skipping manipulation affected behaviour in a multi-round PGG with and without the possibility of punishment. The overall pattern of group contributions over time was subtly, though statistically significantly, different between the two conditions. More detailed analysis showed that participants' decision-making processes differed in multiple ways that cannot be neatly summarised as hungry people being 'more' or 'less' cooperative overall. In the no-punishment game, participants who had skipped breakfast started out by contributing significantly more in the first round. Thereafter, the no-breakfast participants were somewhat more influenced by the contributions of the other group members in the previous round than the breakfast participants were. Specifically, the no-breakfast participants came closer to matching what their group-mates had on average contributed in the previous round when those contributions were high; the breakfast participants fell quite far short of this golden rule (Fig. 2C). In the punishment game, nobreakfast participants were significantly less likely to avail themselves of the option to punish. The consequence was that the availability of punishment was, for the no-breakfast groups, less effective at increasing contributions compared to those made in the no-punishment game.

The increased contributions at the beginning of the no-punishment game, and the greater propensity to match the previous contributions of others, in the nobreakfast groups could be interpreted in terms of the hungry participants thinking less thoroughly and deliberately about the strategic ramifications of their decision (Orquin and Kurzban 2016; Rantapuska et al. 2017). This would be an example of a 'social bright side' to limited cognitive processing, similar to what Halali, Bereby-Meyer and Meiran (2014) observed using cognitive depletion paradigms in trust games. In line with this, the effect of experimental condition on initial contributions was in the same (positive) direction as the effect of getting more of the comprehension questions wrong (although nobreakfast participants did not get significantly more comprehension questions wrong). Moreover, no-breakfast participants made their contribution decisions faster than breakfast participants in the no-punishment game, although faster decisions did not lead to higher contrbutions overall in this game.

The lessened effectiveness of the availability of punishment in increasing contribution levels in the no-breakfast groups was not because no-breakfast participants responded differently to breakfast participants when they were punished. Rather, it was because participants in the no-breakfast groups were significantly less likely to take up the option of punishing their group-mates. As we stressed in the General Introduction, punitiveness was a variable for which predictions in either direction were possible. On the one hand, hunger can increase aggression (DeWall et al. 2011; Bushman et al. 2014), and previous studies have shown that other state manipulations, such as time pressure, lack of sleep or ego depletion tend to increase punitiveness (Sutter et al. 2003; Anderson and Dickinson 2010; Halali et al. 2014). On the other hand, two recent studies found that hunger reduced moral disapproval (Vicario et al. 2018) and the severity of suggested punishments (Kerry et al. 2019). Our punishment finding is in line with the latter results. 


\section{Comparison of Experiments}

Our two experiments present apparently mixed results: no significant condition effects in experiment 1 , and three different significant effects of condition in experiment 2. As we stressed in the General Introduction, the two paradigms used differ in their interaction richness and the pathways to the evolution or demise of cooperation that they allow the experimenter to measure. Nonetheless, some parameters of the two experiments can be compared to one another. For example, both experiments contain a measure of initial generosity: the proposer's offer in the UG, and the first-round no punishment game contribution in the PGG. These are not identical; for example, in the UG the proposer may be anticipating the risk of a rejection in deciding on their offer, which is not applicable to the PGG. Nonetheless, it may be worthwhile comparing these two variables. A second pair of comparable parameters is to do with punitiveness: the responder's minimum acceptable offer in the UG, and the propensity to punish in the PGG. Both involve reducing the payoff of another player at cost to self. Again, the analogy is not perfect: in the PGG but not UG, the relevant behaviour is specifically described as punishment. Thus, for two of the three significant effects in experiment 2, there was a parameter of experiment 1 that was arguably comparable. The third significant effect, the extent to which players' contributions in a round were influenced by the other players' contributions in the previous round of the no-punishment game, had no counterpart in experiment 1.

For the significant findings of experiment 2 , we therefore systematically compared the parameter estimates across the two experiments where possible (Fig. 4). Where there were arguably comparable measures across the two experiments, we combined them using a random-effects meta-analysis (for methods information see Fig. 4 caption). Precision of parameter estimation was lower for experiment 1 due to the smaller sample size. The significantly greater generosity of no-breakfast participants in the first round of experiment 2 , and the non-significant trend in the same direction of experiment 1 , combined to give a significant positive effect overall $(\mathrm{B}=0.24$, s.e. 0.12 , $\mathrm{z}=2.00, p=0.045)$. The significantly reduced punitiveness of no-breakfast participants in experiment 2 combined with a non-significant trend in the opposite direction in

Exp. 1 proposer
Exp. 2. First round, no pun.
Exp. 2. Response to others, no pun.

Fig. 4 Comparison of significant experimental effects from experiment 2 to analogous parameters from experiment 1 where applicable. Points and braces represent parameter estimates and their 95\% confidence intervals. Contributions were standardized to facilitate comparison across studies. Diamonds represent combined meta-analytic estimates and their $95 \%$ confidence intervals. Meta-analytic estimation used random effects estimated by reduced maximum likelihood using R package 'metafor' 
experiment 1 , to give a null effect overall $(\mathrm{B}=-0.04$, s.e. $0.18, \mathrm{z}=-0.20, p=0.85)$. Thus, to the extent that we can consider the two experiments comparable, and for the cases where analogous parameters are measured in both, the overall evidence supports great initial generosity of hungry people, but the evidence on reduced punitiveness is mixed. The greater influence of what the other players are doing on hungry people is only estimated in experiment 2 .

\section{General Discussion}

We have presented two experiments on the effects of acute hunger on cooperation processes. Experiment 1 used a single-shot UG game and, replicating an earlier study, found no effects of participants having skipped breakfast. Experiment 2 went beyond the existing literature and used a task featuring repeated interactions between the same players, and the explicit possibility of punishment. Here, we found systematic differences according to whether group members had skipped breakfast or not. The differences were complex.

First, no-breakfast participants started out in the no-punishment game by contributing more to the common fund. Second, in subsequent rounds, they were more influenced in their contributions by what their group-mates had contributed in the previous round. Specifically, no-breakfast participants came closer to matching high contributions that their group-mates had made, whereas breakfast participants fell well short of this. This meant that as long as the other members had made high contributions initially, high levels of contribution were maintained in the no-breakfast groups. This appears to be an example of a 'social bright side' of limited elaborate processing of the situation (Halali et al. 2014; Rand 2016; Rantapuska et al. 2017), given that nobreakfast participants also spent less time thinking about their contributions in the no-punishment game. This may have meant they were less likely to realise that noncontribution is the income-maximising strategy in the PGG without punishment, regardless of what other group members do. It is also consistent with a hypothesis suggested by Nettle (2019), that hunger will produce a shift away from expensive individual cognitive effort, and towards the less effortful imitation of others.

Third, the availability of punishment was less effective at boosting contributions in the no-breakfast groups than in the breakfast groups. This was explained by nobreakfast participants making less use of the punishment option for a given level of contribution by their group mates, particularly at high contribution levels. The finding of reduced punitiveness is consistent with the results of recent experimental studies of the effects of hunger on moral disapproval (Vicario et al. 2018) and punitive sentiment (Kerry et al. 2019).

Do the findings of our experiment 2 support the lay belief that hunger undermines social cooperation? Certainly, they suggest that once experimental tasks are made rich enough to incorporate more complex interactional dynamics between people, effects of hunger that are not visible using a simple DG-style task may be seen (though these effects require replication, especially since the punitiveness effect observed in experiment 2 was not seen in the responder role of experiment 1). However, the findings of experiment 2 cannot simply be summarized as hunger making people 'less' cooperative; and indeed, contributions did not break down in 
the no-breakfast groups of experiment 2 to a markedly greater extent than in the breakfast groups. The greater matching of group-mates' previous high contributions that we saw in the no-breakfast groups could lead cooperation to persist for longer when people are hungry, contrary to the lay belief. On the other hand, the reduced punitiveness of hungry people could lead to lower cooperation in real-world settings, specifically in those situations where effective punishment is what sustains the cooperative effort.

Our findings, though tentative, do at least suggest a clear avenue for future research, namely exploring hunger effects on cooperation using richer tasks that capture more of the diverse pathways by which real-world cooperation is maintained or fails. It would be beneficial in future to measure emotional reactions and perceptions at the same time as game decisions. For example, one possible emotional mediator of the reduced punitiveness of hungry people is the reduced disgust seen when people are hungry (Vicario et al. 2018; Kerry et al. 2019), but neither our study nor the two early ones measured disgust directly.

If the effects of hunger on cooperation-related decisions that we observed in experiment 2 prove robust, this does have real-world implications for social inequalities in cooperation and anti-social behaviour. The most deprived communities in affluent countries such as the USA and UK feature surprisingly high levels of hunger and food insecurity (Weinreb et al. 2002; Gundersen et al. 2011; Nettle 2017). These same communities also often feature high crime rates, low trust, and low expectations that others will cooperate (Sampson et al. 1997; Nettle et al. 2011; Holland et al. 2012; Schroeder et al. 2014). In particular, they tend to be characterised by low 'collective efficacy', that is, unwillingness of people to intervene to uphold norms of behaviour (Sampson et al. 1997). Low collective efficacy is reminiscent of the reduced punitiveness under hunger that we observed in experiment 2, and was also seen in previous studies (Vicario et al. 2018; Kerry et al. 2019). On the other hand, observers often experience deprived communities as having greater immediate friendliness and sociability (see Hill et al. 2014; Nettle 2015), which is reminiscent of our higher contribution level in the initial round of the no-punishment game. Thus, the diverse effects we have observed, if they prove replicable, could be relevant to understanding how different communities can have such divergent outcomes when it comes to pro- and anti-social behaviour.

Author Contributions Both authors developed hypotheses and protocol design, as well as analysing results collaboratively. SF was responsible for conducting data collection and manuscript production and editing. DN produced and edited the manuscript and interpreted data.

Funding This project has received funding from the European Research Council (ERC) under the European Union's Horizon 2020 research and innovation programme (grant agreement No AdG 666669, COMSTAR)

Data Availability The datasets and code generated for this study can be found in the following Zenodo repository: https://zenodo.org/record/3469364

\section{Compliance with Ethical Standards}

Conflict of Interest The authors declare that the research was conducted in the absence of any commercial or financial relationships that could be construed as a potential conflict of interest. 


\section{Appendix}

Table 1 Full model output for experiment 2

\begin{tabular}{|c|c|c|c|c|c|c|}
\hline Model & Data & Outcome & Predictor & B (s.e.) & $\mathrm{t}$ or $\mathrm{z}$ & $p$ value \\
\hline \multirow[t]{7}{*}{1} & \multirow[t]{7}{*}{ All } & \multirow[t]{7}{*}{ Contribution } & Previous rounds & $-0.49(0.05)$ & -10.25 & $<0.001$ \\
\hline & & & Game & $1.54(0.36)$ & 4.28 & $<0.001$ \\
\hline & & & Condition & $1.96(0.90)$ & 2.17 & 0.033 \\
\hline & & & Previous rounds $*$ Game & $0.69(0.07)$ & 10.15 & $<0.001$ \\
\hline & & & Previous rounds $*$ Condition & $-0.12(0.07)$ & -1.76 & 0.079 \\
\hline & & & Game $*$ Condition & $-1.42(0.52)$ & -2.75 & 0.006 \\
\hline & & & $\begin{array}{l}\text { Previous rounds } * \\
\text { Game } * \text { Condition }\end{array}$ & $0.18(0.10)$ & 1.83 & 0.067 \\
\hline 2 & $\begin{array}{l}\text { No-punishment game, } \\
\text { first round }\end{array}$ & Contribution & Condition & $1.94(0.73)$ & 2.66 & 0.008 \\
\hline \multirow[t]{4}{*}{3} & \multirow[t]{4}{*}{$\begin{array}{l}\text { No-punishment game, } \\
\text { rounds } 2-10\end{array}$} & \multirow[t]{4}{*}{ Contribution } & $\begin{array}{l}\text { Lagged own } \\
\text { contribution }\end{array}$ & $0.41(0.02)$ & 5.36 & $<0.001$ \\
\hline & & & $\begin{array}{l}\text { Lagged mean } \\
\quad \text { contribution of others }\end{array}$ & $0.24(0.04)$ & 6.03 & $<0.001$ \\
\hline & & & Condition & $-0.83(0.49)$ & -1.67 & 0.096 \\
\hline & & & $\begin{array}{l}\text { Lagged mean } \\
\text { contribution of } \\
\text { others*Condition }\end{array}$ & $0.14(0.05)$ & 2.70 & 0.007 \\
\hline \multirow[t]{3}{*}{4} & \multirow[t]{3}{*}{ Punishment game } & \multirow[t]{3}{*}{$\begin{array}{l}\text { Decision to } \\
\text { punish }\end{array}$} & $\begin{array}{l}\text { Mean contribution } \\
\text { of others }\end{array}$ & $-0.31(0.11)$ & -2.88 & 0.004 \\
\hline & & & Condition & $-0.41(0.35)$ & -1.17 & 0.243 \\
\hline & & & $\begin{array}{l}\text { Mean contribution } \\
\text { of others } * \text { Condition }\end{array}$ & $-0.52(0.16)$ & -3.14 & 0.002 \\
\hline \multirow[t]{7}{*}{5} & \multirow[t]{7}{*}{ Punishment game } & \multirow[t]{7}{*}{$\begin{array}{l}\text { Response to } \\
\text { punishment }\end{array}$} & Lagged contribution & $0.45(0.05)$ & 9.93 & $<0.001$ \\
\hline & & & Condition & $0.57(1.18)$ & 0.48 & 0.630 \\
\hline & & & Lagged punishment & $2.17(0.68)$ & 3.20 & 0.001 \\
\hline & & & $\begin{array}{l}\text { Lagged contribution * } \\
\text { Condition }\end{array}$ & $-0.01(0.06)$ & -0.09 & 0.929 \\
\hline & & & $\begin{array}{l}\text { Lagged contribution * } \\
\text { Lagged punishment }\end{array}$ & $-0.17(0.05)$ & -3.36 & $<0.001$ \\
\hline & & & $\begin{array}{l}\text { Condition * Lagged } \\
\text { punishment }\end{array}$ & $-0.06(0.96)$ & -0.06 & 0.954 \\
\hline & & & $\begin{array}{l}\text { Lagged contribution * } \\
\text { Condition * Lagged } \\
\text { punishment }\end{array}$ & $0.02(0.07)$ & 0.23 & 0.820 \\
\hline \multirow[t]{5}{*}{6} & \multirow[t]{5}{*}{ All } & \multirow[t]{5}{*}{$\begin{array}{l}\text { Log latency } \\
\text { to decide } \\
\text { contribution }\end{array}$} & Previous rounds & $-0.13(0.01)$ & -21.43 & $<0.001$ \\
\hline & & & Game & $-0.03(0.05)$ & -0.55 & 0.583 \\
\hline & & & Condition & $-0.21(0.06)$ & -3.62 & $<0.001$ \\
\hline & & & Previous rounds $*$ Game & $0.02(0.009)$ & 2.27 & 0.023 \\
\hline & & & Previous rounds $*$ Condition & $0.01(0.01)$ & 1.53 & 0.127 \\
\hline
\end{tabular}


Table 1 (continued)

\begin{tabular}{|c|c|c|c|c|c|c|}
\hline Model & Data & Outcome & Predictor & B (s.e.) & $\mathrm{t}$ or $\mathrm{z}$ & $p$ value \\
\hline \multirow{5}{*}{7} & \multirow{5}{*}{ Punishment game } & \multirow{5}{*}{$\begin{array}{l}\text { Log latency } \\
\text { to decide } \\
\text { punishment }\end{array}$} & Game $*$ Condition & $0.30(0.07)$ & 4.52 & $<0.001$ \\
\hline & & & $\begin{array}{l}\text { Previous rounds } * \\
\text { Game * Condition }\end{array}$ & $-0.02(0.01)$ & -1.25 & 0.21 \\
\hline & & & Previous rounds & $-0.13(0.01)$ & -23.98 & $<0.001$ \\
\hline & & & Condition & $-0.03(0.08)$ & -0.42 & 0.67 \\
\hline & & & Previous rounds $*$ Condition & $0.01(0.01)$ & 0.97 & 0.34 \\
\hline
\end{tabular}

All models contain random effects of participant nested within group, except for model 2. Model 4 is a generalized linear mixed model with a binomial error structure. All other models are general linear mixed models. The reference categories are no punishment for Game and breakfast for Condition. N=264 (116 in the breakfast condition, 148 in the no-breakfast condition)

Open Access This article is licensed under a Creative Commons Attribution 4.0 International License, which permits use, sharing, adaptation, distribution and reproduction in any medium or format, as long as you give appropriate credit to the original author(s) and the source, provide a link to the Creative Commons licence, and indicate if changes were made. The images or other third party material in this article are included in the article's Creative Commons licence, unless indicated otherwise in a credit line to the material. If material is not included in the article's Creative Commons licence and your intended use is not permitted by statutory regulation or exceeds the permitted use, you will need to obtain permission directly from the copyright holder. To view a copy of this licence, visit http://creativecommons.org/licenses/by/4.0/.

\section{References}

Allen, C., \& Nettle, D. (2019). Hunger and socioeconomic impulsivity in humans background additively predict impulsivity in humans. Current Psychology. https://doi.org/10.1007/s12144-019-0141-7.

Anderson, C., \& Dickinson, D. L. (2010). Bargaining and trust: The effects of 36-h total sleep deprivation on socially interactive decisions. Journal of Sleep Research, 19, 54-63. https://doi.org/10.1111/j.13652869.2009.00767.x.

Arnold, D. (1993). Social crisis and epidemic disease in the famines of nineteenth century India. Social History of Medicine, 6, 385-404.

Boyd, R., \& Richerson, P. J. (1992). Punishment allows the evolution of cooperation (or anything else) in sizable groups. Ethology and Sociobiology, 13, 171-195. https://doi.org/10.1016/0162-3095(92)90032-Y.

Briers, B., Pandalaere, M., Dewitte, S., \& Warlop, L. (2006). Hungry for money. Psychological Science, 85, 20-21. https://doi.org/10.1111/j.1467-9280.2006.01808.x.

Bushman, B. J., DeWall, C. N., Pond, R. S., \& Hanus, M. D. (2014). Low glucose relates to greater aggression in married couples. Proceedings of the National Academy of Sciences, 111, 6254-6257. https://doi. org/10.1073/pnas.1400619111.

Chen, D. L., Schonger, M., \& Wickens, C. (2016). oTree-an open-source platform for laboratory, online, and field experiments. Journal of Behavioral and Experimental Finance, 9, 88-97. https://doi.org/10.1016/j. jbef.2015.12.001.

DeWall, C. N., Deckman, T., Gaillot, M. T., \& Bushman, B. J. (2011). Sweetened blood cools hot tempers: Physiological self-control and aggression. Aggressive Behavior, 37, 73-80. https://doi.org/10.1038 /jid.2014.371.

Dirks, R. (1980). Social responses during severe food shortages and famine [and comments and reply]. Current Anthropology, 21, 21-44. https://doi.org/10.1086/202399. 
Faul, F., Erdfelder, E., Lang, A. G., \& Buchner, A. (2007). G*power 3: A flexible statistical power analysis program for the social, behavioral, and biomedical sciences. Behavior Research Methods, 39, 175-191. https://doi.org/10.3758/BF03193146.

Fehr, E., \& Gachter, S. (2000). Cooperation and punishment in public goods experiments. The American Economic Review, 90, 980-994.

Fehr, E., \& Gächter, S. (2002). Altruistic punishment in humans. Nature, 415, 137-140. https://doi. org/10.1038/415137a.

Frederick, S. (2005). Cognitive reflection and decision making. The Journal of Economic Perspectives, 19, 25-42. https://doi.org/10.1257/089533005775196732.

Gundersen, C., Kreider, B., \& Pepper, J. (2011). The economics of food insecurity in the United States. Applied Economic Perspectives and Policy, 33, 281-303. https://doi.org/10.1093/aepp/ppr022.

Gunnthorsdottir, A., Houser, D., \& McCabe, K. (2007). Disposition, history and contributions in public goods experiments. Journal of Economic Behavior and Organization, 62, 304-315. https://doi.org/10.1016/j. jebo.2005.03.008.

Guth, W., Schmittberger, R., \& Schwarze, B. (1982). An experimental analysis of ultimatum bargaining. Journal of Economic Behavior and Organization, 3, 367-388.

Halali, E., Bereby-Meyer, Y., \& Meiran, N. (2014). Between self-interest and reciprocity: The social bright side of self-control failure. Journal of Experimental Psychology. General, 143, 745-754. https://doi. org/10.1037/a0033824.

Harel, I., \& Kogut, T. (2015). Visceral needs and donation decisions: Do people identify with suffering or with relief? Journal of Experimental Social Psychology, 56, 24-29. https://doi.org/10.1016/j.jesp.2014.08.005.

Häusser, J. A., Stahlecker, C., Mojzisch, A., Leder, J., Van Lange, P. A. M., \& Faber, N. S. (2019). Acute hunger does not always undermine prosociality. Nature Communications, 10, 1-10. https://oi. org/10.1038/s41467-019-12579-7.

Hill, J., Jobling, R., Pollet, T. V., \& Nettle, D. (2014). Social capital across urban neighborhoods: A comparison of self-report and observational data. Evolutionary Behavioral Sciences, 8, 59-69. https://doi.org/10.1037/h0099131.

Holland, J., Silva, A. S., \& Mace, R. (2012). Lost letter measure of variation in altruistic behaviour in 20 neighbourhoods. PLoS One, 7, 1-4. https://doi.org/10.1371/journal.pone.0043294.

Kerry, N., Loria, R. N., \& Murray, D. R. (2019). Gluttons for punishment? Experimentally induced hunger unexpectedly reduces harshness of suggested punishments. Adaptive Human Behavior and Physiology, 5 , 352-370. https://doi.org/10.1007/s40750-019-00121-4.

Nettle, D. (2015). Tyneside Neighbourhoods: Deprivation, social life and social behaviour in one British City. Cambridge: OpenBook Publishers.

Nettle, D. (2017). Does hunger contribute to socioeconomic gradients in behavior? Frontiers in Psychology, 8 , 358. https://doi.org/10.3389/fpsyg.2017.00358.

Nettle, D. (2019). State-dependent cognition and its relevance to cultural evolution. Behavioural Processes, 161, 101-107. https://doi.org/10.1016/j.beproc.2018.01.018.

Nettle, D., Colléony, A., and Cockerill, M. (2011). Variation in cooperative behaviour within a Single City. PLoS One 6, e26922. Doi:Doi:10.1371/ journal.Pone.0026922.

Oosterbeek, H., Sloof, R., \& Van De Kuilen, G. (2004). Cultural differences in ultimatum game experiments: Evidence from a meta-analysis. Experimental Economics, 7, 171-188. https://doi.org/10.1023 /B:EXEC.0000026978.14316.74.

Orquin, J. L., \& Kurzban, R. (2016). A meta-analysis of blood glucose effects on human decision making. Psychological Bulletin, 142, 546-567. https://doi.org/10.1037/bul0000035.

R Core Development Team (2018). R: A Language and Environment for Statistical Computing.

Raihani, N. J., \& Bshary, R. (2019). Punishment: One tool, many uses. Evol. Hum. Sci., 1, e12. https://doi. org/10.1017/ehs.2019.12.

Rand, D. G. (2016). Cooperation, fast and slow: Meta-analytic evidence for a theory of social heuristics and self-interested deliberation. Psychological Science, 27, 1192-1206. https://doi.org/10.1177 $/ 0956797616654455$.

Rantapuska, E., Freese, R., Jääskeläinen, I. P., \& Hytönen, K. (2017). Does short-term hunger increase trust and trustworthiness in a high trust society? Frontiers in Psychology, 8, 1-13. https://doi.org/10.3389 /fpsyg.2017.01944.

Richards, A. (1932). Hunger and work in a savage tribe. London: Routledge.

Sampson, R. J., Raudenbush, S. W., and Earls, F. (1997). Neighborhoods and violent crime: A multilevel study of collective efficacy. Science (80-. ). 277, 918-924. 
Schroeder, K. B., Pepper, G. V, and Nettle, D. (2014). Local norms of cheating and the cultural evolution of crime and punishment : A study of two urban neighborhoods. PeerJ 2, e450. doi:https://doi.org/10.7717 /peerj.450.

Sutter, M., Kocher, M., \& Strauß, S. (2003). Bargaining under time pressure in an experimental ultimatum game. Economics Letters, 81, 341-347. https://doi.org/10.1016/S0165-1765(03)00215-5.

Tisserand, J.-C., Cochard, F., \& le Gallo, J. (2015). Altruistic or strategic considerations : A meta-analysis on the ultimatum and dictator games. In Besancon Available at: http://metaanalysis2014.econ.uoa. gr/fileadmin/metaanalysis2014.econ.uoa.gr/uploads/Tisserand_Jean-Christian.pdf.

Toplak, M. E., West, R. F., \& Stanovich, K. E. (2014). Assessing miserly information processing: An expansion of the cognitive reflection test. Thinking and Reasoning, 20, 147-168. https://doi. org/10.1080/13546783.2013.844729.

Trouche, E., Sander, E., \& Mercier, H. (2014). Arguments, more than confidence, explain the good performance of reasoning groups. Journal of Experimental Psychology. General, 143, 1958-1971. https://doi.org/10.1037/a0037099.

Turnbull, C. M. (1972). The mountain people. London: Jonathan Cape.

Vicario, C. M., Kuran, K. A., Rogers, R., \& Rafal, R. D. (2018). The effect of hunger and satiety in the judgment of ethical violations. Brain and Cognition, 125, 32-36. https://doi.org/10.1016/j. bandc.2018.05.003.

Weinreb, L., Wehler, C., Perloff, J., Scott, R., Hosmer, D., Sagor, L., et al. (2002). Hunger: Its impact on children's health and mental health. Pediatrics, 110, 1-9.

Xu, H., Bègue, L., Sauve, L., \& Bushman, B. J. (2014). Sweetened blood sweetens behavior. Ego depletion, glucose, guilt, and prosocial behavior. Appetite, 81, 8-11. https://doi.org/10.1016/j.appet.2014.05.023.

Publisher's Note Springer Nature remains neutral with regard to jurisdictional claims in published maps and institutional affiliations.

\section{Affiliations}

\section{Sam Fraser ${ }^{1} \cdot$ Daniel Nettle $^{1}$}

1 Population Health Sciences Institute, Newcastle University, Newcastle, UK 